PROCEEDINGS OF THE

AMERICAN MATHEMATICAL SOCIETY

Volume 139, Number 6, June 2011, Pages 2007-2008

S 0002-9939(2010)10546-4

Article electronically published on November 2, 2010

\title{
A FORMULA FOR THE DISCRIMINANT OF NUMBER FIELDS
}

\author{
PEI-CHU HU AND ZHUAN YE
}

(Communicated by Ken Ono)

\begin{abstract}
We obtain a formula for the discriminant $D_{\kappa / \mathbb{Q}}$ of an algebraic number field $\kappa$ in terms of a ratio of the first two coefficients of the Taylor series of $\zeta_{\kappa}$ at $1 / 2$.
\end{abstract}

Let $\kappa$ be a number field of degree $n=r_{1}+2 r_{2}$, where $r_{1}, r_{2}$ are the number of real, complex places respectively. The Dedekind $\zeta$-function of the number field $\kappa$ is defined by the series

$$
\zeta_{\kappa}(s)=\sum_{\mathfrak{a}} \frac{1}{\mathcal{N}(\mathfrak{a})^{s}},
$$

where $\mathfrak{a}$ varies over the non-zero integral ideals of $\kappa$ and $\mathcal{N}(\mathfrak{a})$ denotes the absolute norm of $\mathfrak{a}$. Denote by $D_{\kappa / \mathbb{Q}}$ the discriminant of $\kappa$.

The Dedekind function $\zeta_{\kappa}(s)$ admits a holomorphic continuation with the exclusion of a simple pole at $s=1$ and satisfies the following functional equation

$$
\zeta_{\kappa}(1-s)=A(s) \zeta_{\kappa}(s) \text {, }
$$

where

$$
A(s)=\left|D_{\kappa / \mathbb{Q}}\right|^{s-\frac{1}{2}}\left(\cos \frac{\pi s}{2}\right)^{r_{1}+r_{2}}\left(\sin \frac{\pi s}{2}\right)^{r_{2}} 2^{(1-s) n} \pi^{-s n} \Gamma^{n}(s) .
$$

A straightforward computation gives $A(1 / 2)=1$. Furthermore, let $\beta_{1, \kappa}(\neq 0), \gamma_{1, \kappa}$ be defined by the Taylor expansion of $\zeta_{\kappa}(s)$ at $s=1 / 2$, i.e.,

$$
\zeta_{\kappa}(s)=\beta_{1, \kappa}\left(s-\frac{1}{2}\right)^{\mu}+\gamma_{1, \kappa}\left(s-\frac{1}{2}\right)^{\mu+1}+\cdots .
$$

It turns out from (11) that $\mu$ is a non-negative even integer. It is well-known ([2]) that

$$
\left|D_{\kappa / \mathbb{Q}}\right|^{1 / n} \geq\left(4 \pi e^{\gamma+1}\right)^{r_{1} / n}\left(4 \pi e^{\gamma}\right)^{2 r_{2} / n}-O\left(n^{-2 / 3}\right),
$$

and further, if the Generalized Riemann Hypothesis is assumed, a much stronger inequality states that

$$
\left|D_{\kappa / \mathbb{Q}}\right|^{1 / n} \geq\left(8 \pi e^{\gamma+\pi / 2}\right)^{r_{1} / n}\left(8 \pi e^{\gamma}\right)^{2 r_{2} / n}-O\left(\log ^{-2} n\right),
$$

where $\gamma$ is the Euler constant. We refer the reader to the survey paper [2] for the history of these bounds. Many mathematicians, such as A. Odlyzko, G. Poitou,

Received by the editors January 4, 2010 and, in revised form, June 9, 2010.

2010 Mathematics Subject Classification. Primary 11R42.

Key words and phrases. Number field, Dedekind $\zeta$-function, discriminant, generalized Riemann hypothesis.

The first author was partially supported by the Natural Science Foundation of China.

(C)2010 American Mathematical Society 
J.-P. Serre and H. Stark, have contributed to this theory. Moreover, explicit forms of these inequalities have proven quite useful in several types of applications in algebraic number theory, as described in [2] for example.

In this short paper, we prove the following theorem.

Theorem. Let $\kappa$ be a number field of degree $n$ and let $D_{\kappa / \mathbb{Q}}, r_{1}, r_{2}, \beta_{1, \kappa}$ and $\gamma_{1, \kappa}$ be defined as above. Then we have

$$
\left|D_{\kappa / \mathbb{Q}}\right|^{1 / n}=\left(8 \pi e^{\gamma+\pi / 2}\right)^{r_{1} / n}\left(8 \pi e^{\gamma}\right)^{2 r_{2} / n} e^{-2 \gamma_{1, \kappa} /\left(n \beta_{1, \kappa}\right)} .
$$

Proof. It is clear from (2) that

$\frac{\zeta_{\kappa}^{\prime}(s)}{\zeta_{\kappa}(s)}=\frac{\mu}{s-\frac{1}{2}}+\frac{\gamma_{1, \kappa}}{\beta_{1, \kappa}}+O\left(\left(s-\frac{1}{2}\right)\right)$ and $\quad-\frac{\zeta_{\kappa}^{\prime}(1-s)}{\zeta_{\kappa}(1-s)}=\frac{\mu}{s-\frac{1}{2}}-\frac{\gamma_{1, \kappa}}{\beta_{1, \kappa}}+O\left(\left(s-\frac{1}{2}\right)\right)$.

Logarithmically differentiating (11) gives

$$
-\frac{\gamma_{1, \kappa}}{\beta_{1, \kappa}}=\log \left|D_{\kappa / \mathbb{Q}}\right|-\frac{r_{1} \pi}{2}-n \log (2 \pi)+n \frac{\Gamma^{\prime}\left(\frac{1}{2}\right)}{\Gamma\left(\frac{1}{2}\right)}+\frac{\gamma_{1, \kappa}}{\beta_{1, \kappa}} .
$$

By using the fact (e.g. [1, page 482) that $\Gamma^{\prime}\left(\frac{1}{2}\right) / \Gamma\left(\frac{1}{2}\right)=-\gamma-\log 4$, we obtain

$$
\frac{\gamma_{1, \kappa}}{\beta_{1, \kappa}}=\frac{n}{2}\{\gamma+\log (8 \pi)\}+\frac{r_{1} \pi}{4}-\frac{1}{2} \log \left|D_{\kappa / \mathbb{Q}}\right|,
$$

which completes the proof.

Clearly, the theorem provides us with a formula to compute the discriminant of individual number fields. Further, it is worth pointing out that the main term in (3) appears exactly in the theorem. Thus, the Serre-Odlyzko inequality (3) can be achieved without assuming the Generalized Riemann Hypothesis if one can give an absolute upper bound for $\gamma_{1, \kappa} / \beta_{1, \kappa}$. Indeed, even a slightly weaker bound on the ratio $\gamma_{1, \kappa} / \beta_{1, \kappa}$ could still improve the unconditional bound.

\section{ACKNOWLEDGMENT}

The authors would like to thank the referees for suggestions that improved the clarity of the manuscript.

\section{REFERENCES}

[1] Hu, P. C. and Yang, C. C., Value distribution theory related to number theory, Birkhäuser, 2006. MR2245631(2008c:32021)

[2] Odlyzko, A. M., Bounds for discriminants and related estimates for class numbers, regulators and zeros of zeta functions: a survey of recent results, Séminaire de Théorie des Nombres, Bordeaux (2) 2 (1990), no. 1, 119-141. MR1061762 (91i:11154)

Department of Mathematics, Shandong University, Jinan 250100, Shandong, People's Republic of China

E-mail address: pchu@sdu.edu.cn

Department of Mathematical Sciences, Northern Illinois University, DeKalb, IlliNOIS 60115

E-mail address: ye@math.niu.edu 